\title{
Educación virtual: incidencia del desempeño docente en el rendimiento académico de los estudiantes universitarios
}

\author{
Edwin Gómez Chipana \\ Universidad Nacional José María Arguedas \\ E-mail: egomez@unajma.edu.pe \\ https://orcid.org/0000-0002-7568-5863
}

\section{RESUMEN}

La investigación que se presenta tuvo como objetivo principal determinar el grado de relación e incidencia del desempeño docente en el rendimiento académico de un grupo de estudiantes universitarios de la Facultad de Ciencias de la Empresa de la Universidad Nacional José María Arguedas, correspondiente al semestre académico 2020-I. Para su realización se evaluó a 60 estudiantes de administración de empresas y 37 estudiantes de contabilidad. En el acopio de los datos del desempeño docente se utilizó la técnica de la encuesta y el instrumento fue el cuestionario estructurado del Desempeño Docente que fue aplicado por la Dirección de Calidad Educativa Universitaria y Capacitación; para los datos del Rendimiento Académico de los estudiantes se tomó en cuenta el Reporte Académico o Actas de Notas emitido por la oficina de Registros Académicos. Los resultados de la investigación fueron procesados por el coeficiente de correlación de Spearman que determinó una correlación positiva baja de $28 \%($ Rho $=0.282)$ entre el desempeño docente y el rendimiento académico de los estudiantes, por lo tanto, en la educación virtual sí hay una incidencia positiva del desempeño docente sobre el rendimiento académico de los estudiantes de la Facultad de Ciencias de la Empresa de la UNAJMA.

Palabras clave: Educación Virtual; Desempeño Docente; Rendimiento Académico; Estudiantes Universitarios 


\title{
Virtual education: incidence of teaching performance on the academic performance of university students
}

\begin{abstract}
The main objective of the research presented was to determine the degree of relationship and incidence of teaching performance in the academic performance of a group of university students from the Faculty of Business Sciences of the José María Arguedas National University, corresponding to the academic semester 2020 -I. To carry it out, 60 business administration students and 37 accounting students were evaluated. The survey technique was used to collect data on teacher performance and the instrument was the structured questionnaire on Teacher Performance, which was applied by the University Educational Quality and Training Department; For the data on the Academic Performance of the students, the Academic Report or Report Cards issued by the Office of Academic Records were taken into account. The research results were processed by the Spearman correlation coefficient, which determined a low positive correlation of $28 \%($ Rho $=0.282)$ between teacher performance and students' academic performance, therefore, in virtual education there is a positive impact of teaching performance on the academic performance of the students of the Faculty of Business Sciences of UNAJMA.'
\end{abstract}

Keywords: Virtual education; Teaching Performance, Academic Performance; University students

Artículo recibido: 03 nov. 2020 Aceptado para publicación: 07 dic. 2020 Correspondenciaegomez@unajma.edu.pe Conflictos de Interés: Ninguna que declarar 


\section{INTRODUCCIÓN}

La Universidad Nacional José María Arguedas (UNAJMA) tiene su origen en la Ley $\mathrm{N}^{\circ} 28372$ del 29 de octubre de 2004, en la actualidad cuenta con 6 escuelas profesionales: Ingeniería Agroindustrial, Ingeniería de Sistemas, Ingeniería Ambiental, Administración de Empresas, Contabilidad y Educación Primaria Intercultural. Hasta el cierre del semestre académico 2020-0 (29-02-2020) las actividades académicas se llevaron con normalidad bajo el régimen de estudios de modalidad presencial de acuerdo al artículo 39 de la Ley Universitaria (Ley N 30220). En el mes de marzo, el Virus Covid-19 llegó para cambiar el escenario educativo tradicional de la formación universitaria, donde la Universidad Nacional José María Arguedas ha tenido que responder con urgencia a esta situación de crisis, teniendo que adaptarse a la educación virtual para cumplir su rol de formador de futuros profesionales en distintos ámbitos de las ciencias del conocimiento humano. La Universidad José María Arguedas comienza a brindar sus servicios educativos virtuales en el semestre 2020-I (15-05-2020), donde el estamento docente empezó a dar soporte a sus actividades educativas, implementando estrategias de educación virtual para el contexto de una educación no presencial al amparo del artículo 39 de la Ley Universitaria (Ley $\mathrm{N}^{\circ}$ 30220). Este traslado del aula tradicional de clases al aula virtual se llevó a cabo como una medida preventiva contra la transmisión del Covid-19 poniendo en expectativa un nuevo modo de enseñar y aprender, donde el estudiante universitario, en la educación virtual, pasa a ser un protagonista indiscutible. La implementación de esta experiencia académica, educación virtual, pone de manifiesto la necesidad de conocer, comprender y resolver los aspectos, tecnológicos y pedagógicos que puedan incidir en la formación profesional de los estudiantes de la Facultad de Ciencias de la Empresa de la UNAJMA. Esta estrategia de educación virtual se está desarrollando mediante la utilización de tecnologías informáticas, donde la didáctica implica cumplir ritmos de aprendizaje del estudiante universitario y modificar el rol del docente universitario, como también, trabajar tendencias educativas tecnológicas y analizar la evaluación en ambientes virtuales del aprendizaje. Las bases pedagógicas de la educación virtual se llevarán a cabo en espacios sincrónicos y asincrónicos y esto implica cambios para docentes y estudiantes que deberán llevar a cabo contenidos, actividades e interacción en un espacio virtual de formación profesional, sin embargo, es importante destacar que realizar clases virtuales no supone haber migrado a un modelo de educación virtual, por lo tanto, es necesario revisar el contexto en que se realiza el desempeño docente y de qué manera incide en el rendimiento académico de los estudiantes de la Facultad de Ciencias de la Empresa de la UNAJMA. La educación virtual también debe 
afianzarse en el contexto de formar profesionales solidarios, con sensibilidad social y adecuadas habilidades y competencias profesionales. La implementación de la educación virtual en la formación profesional de Los estudiantes de la Facultad de Ciencias de la Empresa no debe ser ajena al cumplimiento de la Ética, la Responsabilidad y el Compromiso Social ya que éstos son los pilares principales que los conduzcan como futuros profesionales y pasen a formar parte directa del desarrollo del Perú. El comportamiento y desempeño profesional de los estudiantes de la Facultad de Ciencias de la Empresa de la UNAJMA, es el resultado de factores educativos y que durante el proceso virtual de formación profesional, también, se trasmitan conocimientos básicos y especializados en base a estándares internacionales que posibilite a que en el futuro, es decir, en el momento en que los estudiantes universitarios salgan de sus aulas ya con una profesión inicien sus actividades profesionales y empresariales sin tropiezos. Se hace indispensable entonces que la Facultad de Ciencias de la Empresa de la UNAJMA, implemente o incorpore buenas prácticas educativas en entornos virtuales en beneficio del estudiante futuro profesional de las ciencias empresariales. En cuanto a los antecedentes de nuestra investigación, Cepeda (2017) en la tesis, Estrategia metodológica del uso de aulas virtuales en el proceso de enseñanza aprendizaje universitario del régimen académico ecuatoriano de la Universidad Nacional de Chimborazo, describe la incidencia de la metodología del aula virtual en la enseñanza aprendizaje de los estudiantes de la carrera de Psicología Educativa del segundo semestre del año 2015 y concluye que la aplicación de la estratégica metodológica de uso de aulas virtuales en el proceso de enseñanza aprendizaje universitario, organizado en función de los cuatro componentes de aprendizaje del reglamento de régimen académico (actividades asistidas por el profesor, las actividades autónomas, las actividades prácticas y actividades de aprendizaje colaborativas) mejoró significativamente en 36 centésimas el promedio general de los estudiantes en la asignatura de Psicología General I. Por otro lado, Cabañas (2003) en la tesis, Aulas virtuales como herramienta de apoyo en la educación de la Universidad Nacional Mayor de San Marcos, describe los aspectos que involucran la implementación de las aulas virtuales como apoyo en la Educación y las bondades que ésta ofrece para los docentes, alumnos y público en general así como los recursos que se necesitan para su implementación y señala como conclusión que el impulso de las nuevas tecnologías en informática y en las comunicaciones están dando un ambiente cambiante a la Educación que a su vez ha recibido una influencia de la cultura del mundo globalizado y que las nuevas tecnologías de información y comunicación propician una nueva forma de aprendizaje, en donde se generan espacios virtuales que faciliten interacciones sociales entre 
los participantes de estos procesos educativos independientemente del tiempo y lugar geográfico donde se encuentren. También, Camarena (2017) en la tesis, Estrategias de enseñanza virtual docente y su influencia en el rendimiento académico de los estudiantes del curso Desempeño Universitario en la Universidad Científica del Sur, describe la relación entre las estrategias de enseñanza virtual y el rendimiento académico de los estudiantes, llegando a la conclusión que no existe relación entre estas variables, excepto en el aula "P" en donde se halló una relación significativa, positiva y débil. Para definir el término educación virtual podemos referirnos a La Universidad Virtual REUNA que define a la educación virtual como aquella modalidad de enseñanza que utiliza redes y computadores para ser impartida, (UNESCO/IESALC, 2003, p. 247). Asimismo, Para el Ministerio de Educación de Colombia, la Educación Virtual se refiere al desarrollo de programas de formación que tienen como escenario de enseñanza y aprendizaje el ciberespacio. No se trata simplemente de una forma singular de hacer llegar la información a lugares distantes, sino que es toda una perspectiva pedagógica, (Mineducación, 2020). Para entender el concepto de desempeño docente podemos referirnos a Montenegro (2007), quien lo define como un conjunto de acciones concretas, como el cumplimiento de sus funciones; éste se halla determinado por factores asociados al propio docente, al estudiante y al entorno (p. 18). Asimismo, Estrada, L. (2013) señala que el desempeño docente es el eje que moviliza el proceso de formación dentro del sistema educativo formal (p. 1). En cuanto al rendimiento académico (Espinoza 2006), cita a Pizarro y Clark (1998, p.18), quienes conciben que el rendimiento académico es una medida de la capacidad de respuesta del individuo, que expresa, en forma estimativa, lo que una persona ha aprendido como resultado de un proceso de instrucción o formación (p. 223). Por otro lado, Reyes (2003) señala que el rendimiento académico es un indicador del nivel de aprendizaje alcanzado por el alumno, por ello, el sistema educativo brinda tanta importancia a dicho indicador. En tal sentido, el rendimiento académico se convierte en una "tabla imaginaria de medida" para el aprendizaje logrado en el aula, que constituye el objetivo central de la educación. Sin embargo, en el rendimiento académico, intervienen muchas otras variables externas al sujeto, como la calidad del maestro, el ambiente de clase, la familia, el programa educativo, etc., y variables psicológicas o internas, como la actitud hacia la asignatura, la inteligencia, la personalidad, el auto concepto del alumno, la motivación, etc. (p. 75). En la actualidad, en la docencia universitaria, se hace importante conocer la relación existente entre el desempeño del docente universitario y el rendimiento académico de los estudiantes universitarios de cualquier profesión. También es importante evaluar la influencia que el desempeño docente tiene en el rendimiento 
académico de los estudiantes universitarios, para así tomar decisiones correctivas que ayuden a mejorar la calidad profesional de los estudiantes universitarios; por tal motivo nos planteamos el problema general de la investigación ¿De qué manera en la educación virtual el desempeño docente incide en el rendimiento académico de los estudiantes de la Facultad de Ciencias de la Empresa de la UNAJMA?; por otro lado, planteamos como posible respuesta a la pregunta la hipótesis general: En la educación virtual el desempeño docente incide positivamente en el rendimiento académico de los estudiantes de la Facultad de Ciencias de la Empresa de la UNAJMA. Una vez efectuado el análisis correspondiente, podremos cumplir con el objetivo general de Conocer la incidencia en la educación virtual del desempeño docente en el rendimiento académico de los estudiantes de la Facultad de Ciencias de la Empresa de la UNAJMA. Cumplir con este objetivo se justifica en la medida que ayudará a tomar decisiones que sean importantes para la mejora continua en bien de la calidad académica del futuro profesional de las ciencias empresariales.

\section{Método y Metodología}

\subsection{General}

La presente investigación tiene un enfoque cuantitativo, ya que es útil para evaluar, comparar, interpretar, establecer precedentes y determinar causalidad y sus implicaciones (Hernández et al., 2014). La información a presentar está referida al desempeño docente en la educación virtual y su incidencia en el rendimiento académico de los estudiantes de la Facultad de Ciencias de la Empresa de la UNAJMA. La investigación tiene un alcance descriptivo, este tipo de estudios tiene como finalidad especificar propiedades y características importantes de cualquier fenómeno que se analice. Describe tendencias de un grupo o población (Hernández et al., 2014). En la determinación de la muestra se aplicó el muestreo no probabilístico o dirigido, según Hernández (2014) significa, “que la elección de los elementos no depende de la probabilidad, sino de causas relacionadas con las características de la investigación o los propósitos del investigador" (p. 176), esto debido a que es un grupo de estudiantes que tienen las mismas características en su formación profesional, que pertenecen al sector de las ciencias empresariales y estudian en la Facultad de Ciencias de la Empresa de la UNAJMA. La investigación es básica, según Carrasco (2005) porque "la investigación que no tiene propósitos aplicativos inmediatos, solo busca ampliar y profundizar el conocimiento que existe a cerca de la realidad" (p. 43). El nivel de investigación es ex post-facto (Diseño no experimental) según Arias (2012) significa posterior al hecho (p. 33). El tipo de estudio para el presente trabajo de investigación es transaccional o transversal, donde lo que hacemos 
es observar fenómenos tal como se dan en su contexto natural, para analizarlos (Hernández et al., 2014). En el presente trabajo de investigación se utilizó, para el acopio de los datos del desempeño docente, la técnica de la encuesta y el instrumento fue el cuestionario estructurado del Desempeño Docente que fue preparado por la Dirección de Calidad Educativa Universitaria y Capacitación de la UNAJMA. Según Carrasco (2005) define a la encuesta "como una técnica de investigación social para la indagación, exploración y recolección de datos, mediante preguntas formuladas directa o indirectamente a los sujetos que constituyen la unidad del análisis del estudio investigativo" (p.314). Para el Rendimiento Académico de los estudiantes se tomó en cuenta el Reporte Académico o Actas de Notas, emitido por la oficina de Registros Académicos de la UNAJMA. Para la presentación, análisis e interpretación de los datos se

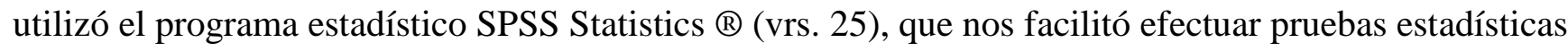
de carácter descriptivo e inferencial entre las variables de estudio desempeño docente y rendimiento académico.

\subsection{Específico}

\section{Con respecto al Diseño del Análisis:}

En la presente investigación se utilizó el enfoque cuantitativo, el método descriptivo y el diseño correlacional unidireccional de Spearman. Para los cálculos se hizo un análisis exploratorio de datos, hallándose lo siguiente:

1. El nivel de significancia equivale a un mínimo de 0.05 (frecuentemente aceptado).

2. En cada tabla de resultados estadísticos inferenciales, hay significancia: $1 *$ un nivel alcanzado a 0.01 y $2 * *$ con significancia de 0.05 .

3. La forma de distribución de los puntajes fue cercanamente normal y fue comprobado verificándolo mediante gráficos y utilizando fórmulas usuales para el cálculo del grado de asimetría y curtosis.

4. La variable independiente cuenta con 10 preguntas relacionadas al Desempeño Docente, en las cuales según revisión estadística cuentan con una confiabilidad para nuestra muestra de $\boldsymbol{\alpha}=\boldsymbol{0 . 9 7 7}$, que representa una consistencia interna alta del instrumento.

5. La variable dependiente está constituida por las notas o calificaciones obtenidas por los estudiantes en su Rendimiento Académico como resultado de su aprendizaje, en las cuales según revisión estadística cuentan con una media de nuestra muestra de 15.71, que representa un calificativo de alto.

\section{Con respecto a los Participantes:}

Población: está constituida por los estudiantes de la Facultad de Ciencias de la Empresa de la UNAJMA. 
Muestra: está constituida por los estudiantes de las Escuelas Profesionales de Administración de Empresas y Contabilidad de la UNAJMA.

Género de los estudiantes: Masculino y Femenino.

Edad: Comprendido en el Rango de 18 a 20 años.

\section{Tabla 1}

\section{Distribución de la muestra según escuela profesional}

\begin{tabular}{|l|l|l|}
\hline Escuela Profesional & $\mathbf{n}$ & \% \\
\hline Administración de Empresas & 60 & 61.86 \\
\hline Contabilidad & 37 & 38.14 \\
\hline Total & $\mathbf{9 7}$ & $\mathbf{1 0 0}$ \\
\hline
\end{tabular}

Fuente: elaboración propia.

Como se puede observar en la tabla 1, el 61.86\% de la población evaluada corresponde a la Escuela Profesional de Administración de Empresas y el 38.14\% corresponde a la Escuela Profesional de Contabilidad.

\section{En cuanto a los Instrumentos:}

- Cuestionario de Desempeño Docente

- Promedio del Rendimiento Académico de los alumnos matriculados en la Facultad de Ciencias de la Empresa: (1) Escuela Profesional de Administración de Empresas (asignaturas Evaluación de Estados Financieros; Costos y presupuestos). (2) Escuela Profesional de Contabilidad (asignaturas Contabilidad III; Ética Profesional del Contador Público).

\section{Resultados y Discusión}

Con respecto a la tabla 2 se puede observar las medias y desviación estándar de las 10 preguntas del cuestionario de Desempeño Docente, en las cuales según revisión estadística cuenta con una media total de nuestra muestra de 3,62 y una desviación estándar total de nuestra muestra de 0,685.

\section{Tabla 2}

\section{Puntajes promedio y desviación estándar para Desempeño Docente}

\begin{tabular}{|l|l|c|c|c|}
\hline Ítem & Preguntas & N & Media & $\begin{array}{l}\text { Desv. } \\
\text { Est. }\end{array}$ \\
\hline $\mathbf{1 .}$ & ¿El docente publica el sílabo en el siga web y socializa su contenido? & 97 & 3,70 &, 766 \\
\hline $\mathbf{2 .}$ & ¿El docente desarrolla la asignatura de acuerdo al sílabo? & 97 & 3,72 &, 703 \\
\hline $\mathbf{3 .}$ & $\begin{array}{l}\text { ¿El docente conoce su asignatura y trasmite adecuadamente sus } \\
\text { conocimientos? }\end{array}$ & 97 & 3,67 &, 688 \\
\hline
\end{tabular}




\begin{tabular}{|l|l|c|c|c|}
\hline 4. & $\begin{array}{l}\text { ¿El docente genera confianza y absuelve las preguntas académicas de los } \\
\text { estudiantes? }\end{array}$ & 97 & 3,38 &, 895 \\
\hline $\mathbf{5 .}$ & $\begin{array}{l}\text { ¿El docente propicia el aprendizaje significativo en las sesiones teóricas o } \\
\text { prácticas? }\end{array}$ & 97 & 3,47 &, 867 \\
\hline $\mathbf{6 .}$ & $\begin{array}{l}\text { ¿El docente utiliza herramientas tecnológicas informáticas para la enseñanza } \\
\text { de la asignatura? }\end{array}$ & 97 & 3,64 &, 695 \\
\hline $\mathbf{7 .}$ & $\begin{array}{l}\text { ¿El docente utiliza estrategias metodológicas (videos, otras) para el proceso } \\
\text { de enseñanza? }\end{array}$ & 97 & 3,75 &, 596 \\
\hline $\mathbf{8 .}$ & ¿El docente inicia y finaliza sus clases según el horario establecido? & 97 & 3,79 &, 594 \\
\hline $\mathbf{9}$ & $\begin{array}{l}\text { ¿El docente devuelve el instrumento de evaluación calificado (exámenes, } \\
\text { otros) para su conocimiento? }\end{array}$ & 97 & 3,66 &, 762 \\
\hline $\mathbf{1 0 .}$ & ¿El docente respeta las opiniones de los estudiantes? & 97 & 3,43 &, 877 \\
\hline N válido (por lista) & 97 & 3,62 &, 685 \\
\hline
\end{tabular}

Fuente: elaboración propia.

En la tabla 3 se puede observar la calificación del Rendimiento Académico de los estudiantes, muestra un mínimo de 10 puntos y un máximo de 19 puntos. Por otro lado, según revisión estadística cuenta con una calificación media total de nuestra muestra de 15,71 y una desviación estándar total de nuestra muestra de $\mathbf{1 , 7 0 9 .}$

Tabla 3

Puntajes promedio y desviación estándar para Rendimiento Académico

\begin{tabular}{|l|l|l|l|l|l|}
\hline \multicolumn{2}{c}{ N Mínimo Máximo } & \multicolumn{3}{c|}{ Media Desv. Est. } \\
\hline Rendimiento Académico & 97 & 10 & 19 & 15,71 & 1,709 \\
\hline N válido (por lista) & 97 & \multicolumn{5}{l|}{} \\
\hline
\end{tabular}

Fuente: elaboración propia.

La tabla 4 nos muestra las correlaciones de las distintas preguntas (ítems) referidas al Desempeño Docente con respecto al Rendimiento Académico de los estudiantes. Se observan estadísticos inferenciales de significancia $1 *$ a un nivel alcanzado a 0,01 (unilateral) y $2 * *$ con significancia de 0,05 (unilateral). Para el Ítem 1 se tiene un coeficiente de correlación de 0,131 y un p-valor =0,101 > 0,05 , esto indica que no hay relación o incidencia del Ítem 1 en el rendimiento académico de los estudiantes. Para el Ítem 2 se tiene un coeficiente de correlación de 0,118 y un p-valor =0,124>0,05, esto indica que no hay relación o incidencia del Ítem 2 en el rendimiento académico de los estudiantes. Para el Ítem 3 se tiene un coeficiente de correlación de 0,155 y un p-valor $=0,065>0,05$, esto indica 
que no hay relación o incidencia del Ítem 3 en el rendimiento académico de los estudiantes. Para el Ítem 4 se tiene un coeficiente de correlación de 0,239 y un p-valor $=0,009<0,05$, esto indica que sí hay una relación o incidencia del Ítem 4 en el rendimiento académico de los estudiantes. Para el Ítem 5 se tiene un coeficiente de correlación de 0,328 y un p-valor $=0,001<0,05$, esto indica que sí hay una relación o incidencia del Ítem 5 en el rendimiento académico de los estudiantes. Para el Ítem 6 se tiene un coeficiente de correlación de 0,223 y un p-valor $=0,014<0,05$, esto indica que sí hay una relación o incidencia del Ítem 6 en el rendimiento académico de los estudiantes. Para el Ítem 7 se tiene un coeficiente de correlación de 0,205 y un p-valor $=0,022<0,05$, esto indica que sí hay una relación o incidencia del Ítem 7 en el rendimiento académico de los estudiantes. Para el Ítem 8 se tiene un coeficiente de correlación de 0,163 y un p-valor $=0,055>0,05$, esto indica que no hay una relación o incidencia del Ítem 8 en el rendimiento académico de los estudiantes. Para el Ítem 9 se tiene un coeficiente de correlación de 0,261 y un p-valor $=0,005<0,05$, esto indica que sí hay una relación o incidencia del Ítem 9 en el rendimiento académico de los estudiantes. Para el Ítem 10 se tiene un coeficiente de correlación de 0,367 y un p-valor $=0,000<0,05$, esto indica que sí hay una relación o incidencia del Ítem 10 en el rendimiento académico de los estudiantes.

Una vez efectuado el análisis estadístico correlacional entre los Ítems del Desempeño Docente y su incidencia con el Rendimiento Académico de los estudiantes, se determinó que los Ítems 1, 2, 3 y 8 tienen un $\mathrm{p}$-valor $=0,101,0,124,0,065 \mathrm{y}$ 0,055 respectivamente, son mayores al 0,05, por lo tanto, podemos afirmar que estos Ítems no tienen relación o incidencia en el Rendimiento Académico de los estudiantes. Entonces, debemos efectuar un ajuste o corrección de los datos estadísticos para determinar una correcta correlación entre el Desempeño Docente y su incidencia en el Rendimiento Académico del estudiante. 
Tabla 4

\section{Correlaciones entre Desempeño Docente y Rendimiento Académico}

\begin{tabular}{|c|c|c|c|c|c|c|c|c|c|c|c|c|c|}
\hline & & & item 1 & Ite & Item 3 & Iten & Item 5 & Iten & em 7 & ítem 8 & ítem 9 & Ítem 10 & Académico \\
\hline \multirow{33}{*}{$\begin{array}{l}\text { Rho de } \\
\text { Spearman }\end{array}$} & \multirow{3}{*}{$\begin{array}{l}\text { ¿El docente publica el sílabo en el siga web y socializa } \\
\text { su contenido? }\end{array}$} & Coeficiente de correlación & 1,000 & $906 "$ & $825^{\prime \prime}$ &, $678^{\prime \prime}$ & ,690" &, $795^{\prime \prime}$ & $934^{\prime \prime}$ & $933^{\prime \prime}$ &, $837^{\prime \prime}$ &, $685^{\prime \prime}$ & 131 \\
\hline & & Sig. (unilateral) & . &, 000 &, 000 &, 000 &, 000 &, 000 &, 000 &, 000 &, 000 &, 000 & ,101 \\
\hline & & $\mathrm{N}$ & 97 & 97 & 97 & 97 & 97 & 97 & 97 & 97 & 97 & 97 & 97 \\
\hline & \multirow{3}{*}{$\begin{array}{l}\text { ¿El docente desarrolla la asignatura de acuerdo al } \\
\text { sílabo? }\end{array}$} & Coeficiente de correlación & $906 "$ & 1,000 &, $794^{\prime \prime}$ & ,671" & $691^{*}$ &, 764 & $903^{* \prime}$ &, $832^{\prime \prime}$ &, $806^{* \prime}$ & ,682" &, 118 \\
\hline & & Sig. (unilateral) &, 000 & . &, 000 &, 000 &, 000 &, 000 &, 000 &, 000 &, 000 &, 000 &, 124 \\
\hline & & $\mathrm{N}$ & 97 & 97 & 97 & 97 & 97 & 97 & 97 & 97 & 97 & 97 & 97 \\
\hline & \multirow{3}{*}{$\begin{array}{l}\text { ¿El docente conoce su asignatura y trasmite } \\
\text { adecuadamente sus conocimientos? }\end{array}$} & Coeficiente de correlación & $825^{\prime \prime}$ &, $794^{*}$ & 1,000 & 807 & $810^{\prime \prime}$ &, $763 "$ &, $763^{\prime \prime}$ &, $772 "$ &, $723^{\prime \prime}$ & $801^{\prime \prime}$ & ,155 \\
\hline & & Sig. (unilateral) &, 000 &, 000 & . &, 000 &, 000 &, 000 & 000 &, 000 &, 000 &, 000 & 065 \\
\hline & & $\mathrm{N}$ & 97 & 97 & 97 & 97 & 97 & 97 & 97 & 97 & 97 & 97 & 97 \\
\hline & \multirow{3}{*}{$\begin{array}{l}\text { ¿El docente genera confianza y absuelve las } \\
\text { preguntas académicas de los estudiantes? }\end{array}$} & Coeficiente de correlación &, $678^{* \prime}$ & ,671" & $807^{* \prime}$ & 1,000 & $934 "$ &, $774^{* \prime}$ &, $693^{*}$ &, $642^{* \prime}$ &, $733^{* \prime}$ & $876^{\prime \prime}$ & $239^{*}$ \\
\hline & & Sig. (unilateral) &, 000 &, 000 &, 000 & . &, 000 &, 000 & 000 &, 000 &, 000 &, 000 &, 009 \\
\hline & & $\mathrm{N}$ & 97 & 97 & 97 & 97 & 97 & 97 & 97 & 97 & 97 & 97 & 97 \\
\hline & \multirow{3}{*}{$\begin{array}{l}\text { ¿El docente propicia el aprendizaje significativo en las } \\
\text { sesiones teóricas o prácticas? }\end{array}$} & Coeficiente de correlación & $690^{\circ \prime}$ & $691^{\prime \prime}$ & $810^{\prime \prime}$ & $934^{* \prime}$ & 1,000 &, $793^{* \prime}$ &, $716^{*}$ &, $657^{* \prime}$ &, $717^{*}$ & $928^{\prime \prime}$ & $328^{* \prime}$ \\
\hline & & Sig. (unilateral) &, 000 &, 000 &, 000 &, 000 & . &, 000 &, 000 &, 000 &, 000 &, 000 &, 001 \\
\hline & & $\mathrm{N}$ & 97 & 97 & 97 & 97 & 97 & 97 & 97 & 97 & 97 & 97 & 97 \\
\hline & \multirow{3}{*}{$\begin{array}{l}\text { ¿El docente utiliza herramientas tecnológicas } \\
\text { informáticas para la enseñanza de la asignatura? }\end{array}$} & Coeficiente de correlación &, $795^{*}$ &, $764^{\prime \prime}$ &, $763^{*}$ &, $774^{*}$ &, $793^{\prime \prime}$ & 1,000 & $841^{\prime \prime}$ &, $738^{\prime \prime}$ &, $822^{* \prime}$ &, $770^{* \prime}$ & $223^{\circ}$ \\
\hline & & Sig. (unilateral) &, 000 &, 000 &, 000 &, 000 &, 000 & . & 000 &, 000 &, 000 &, 000 &, 014 \\
\hline & & $\mathrm{N}$ & 97 & 97 & 97 & 97 & 97 & 97 & 97 & 97 & 97 & 97 & 97 \\
\hline & \multirow{3}{*}{$\begin{array}{l}\text { ¿El docente utiliza estrategias metodológicas (videos, } \\
\text { otras) para el proceso de enseñanza? }\end{array}$} & Coeficiente de correlación & $934^{\prime \prime}$ & $903^{* \prime}$ &, $763^{\prime \prime}$ & ,693" &, $716^{*}$ &, $841^{\prime \prime}$ & 1,000 &, $872^{* \prime}$ &, $890^{* \prime}$ & ,706" & $205^{\circ}$ \\
\hline & & Sig. (unilateral) &, 000 &, 000 &, 000 &, 000 &, 000 &, 000 & $\cdot$ &, 000 &, 000 &, 000 &, 022 \\
\hline & & $\mathrm{N}$ & 97 & 97 & 97 & 97 & 97 & 97 & 97 & 97 & 97 & 97 & 97 \\
\hline & \multirow{3}{*}{$\begin{array}{l}\text { ¿El docente inicia y finaliza sus clases según el horario } \\
\text { establecido? }\end{array}$} & Coeficiente de correlación & $933^{* \prime}$ & $832^{\prime \prime}$ &, $772^{\prime \prime}$ & ,642" & $657^{* \prime}$ &, $738^{* \prime}$ &, $872^{* \prime}$ & 1,000 &, $831^{* \prime}$ & $660^{\circ \prime}$ & , 163 \\
\hline & & Sig. (unilateral) &, 000 &, 000 &, 000 &, 000 &, 000 &, 000 &, 000 & . &, 000 &, 000 &, 055 \\
\hline & & $\mathrm{N}$ & 97 & 97 & 97 & 97 & 97 & 97 & 97 & 97 & 97 & 97 & 97 \\
\hline & \multirow{3}{*}{$\begin{array}{l}\text { ¿El docente devuelve el instrumento de evaluación } \\
\text { calificado (exámenes, otros) para su conocimiento? }\end{array}$} & Coeficiente de correlación & $837^{\prime \prime}$ & $806^{*}$ &, $723^{\prime \prime}$ &, $733^{*}$ &, $717^{* \prime}$ &, $822^{* \prime}$ &, $890^{\circ \prime}$ &, $831^{\prime \prime}$ & 1,000 & ,707" & $261^{*}$ \\
\hline & & Sig. (unilateral) &, 000 &, 000 &, 000 &, 000 &, 000 &, 000 & 000 &, 000 & . &, 000 &, 005 \\
\hline & & $\mathrm{N}$ & 97 & 97 & 97 & 97 & 97 & 97 & 97 & 97 & 97 & 97 & 97 \\
\hline & \multirow[t]{3}{*}{ ¿El docente respeta las opiniones de los estudiantes? } & Coeficiente de correlación &, $685^{*}$ & $682^{\prime \prime}$ & $801 "$ & $876^{*}$ & $928^{\prime \prime}$ &, $770 \%$ &, $706^{*}$ &, $660^{\circ \prime}$ &, $707^{* 1}$ & 1,000 & $367^{* \prime}$ \\
\hline & & Sig. (unilateral) &, 000 &, 000 &, 000 &, 000 &, 000 &, 000 & ,000 &, 000 &, 000 & . &, 000 \\
\hline & & $\mathrm{N}$ & 97 & 97 & 97 & 97 & 97 & 97 & 97 & 97 & 97 & 97 & 97 \\
\hline & \multirow[t]{3}{*}{ Rendimiento Académico } & Coeficiente de correlación &, 131 &, 118 &, 155 & , $239^{* \prime}$ & $328^{\prime \prime}$ & $223^{*}$ & $205^{\circ}$ & 163 &, $261^{*}$ & $367^{* \prime}$ & 1,000 \\
\hline & & Sig. (unilateral) & 101 & 124 &, 065 & 009 & 001 & 014 & 022 & 055 &, 005 &, 000 & \\
\hline & & $\overline{\mathrm{N}}$ & 97 & 97 & 97 & 97 & 97 & 97 & 97 & 97 & 97 & 97 & 97 \\
\hline
\end{tabular}

"*. La correlación es significativa en el nivel 0,01 (unilateral).

*. La correlación es significativa en el nivel 0,05 (unilateral).

Fuente: elaboración propia.

En la tabla 5 podemos observar el promedio y desviación estándar (corregido) para Desempeño Docente, sin tomar en cuenta los ítems 1,2,3 y 8, obteniéndose una calificación media total de nuestra muestra de 3,56 y una desviación estándar total de nuestra muestra de $\mathbf{0 , 7 2 5}$.

En cuanto a Rendimiento Académico de los estudiantes, éste sigue manteniendo la media total de nuestra muestra de 15,71 y una desviación estándar total de nuestra muestra de 1,709. 


\section{Tabla 5}

Puntajes promedio y desviación estándar

\begin{tabular}{|c|c|c|c|c|c|}
\hline & $\mathrm{N}$ & Mínimo & Máximo & Media & Desv. Desviación \\
\hline Desempeño Docente & 97 & 1 & 4 & 3,56 &, 725 \\
\hline Rendimiento Académico & 97 & 10 & 19 & 15,71 & 1,709 \\
\hline N válido (por lista) & 97 & & & & \\
\hline
\end{tabular}

Fuente: elaboración propia.

Con respecto a la tabla 6 podemos observar el coeficiente o grado de correlación de Spearman entre el desempeño Docente y el Rendimiento Académico de los estudiantes, habiéndose determinado una Correlación Positiva Baja de $\boldsymbol{R h o}=\mathbf{0 . 2 8 2}$, es decir, que existe una asociación Positiva Baja de $28 \%$ entre el Desempeño Docente y el Rendimiento Académico de los estudiantes. Por otro lado, también podemos observar que el p-valor $=0,003<0,05$ y es significativa en el nivel 0,01(unilateral), esto indica que sí hay una relación o incidencia positiva del Desempeño Docente sobre el Rendimiento Académico de los estudiantes

\section{Tabla 6}

\section{Coeficiente de Correlación}

\begin{tabular}{|c|c|c|c|c|}
\hline & & & $\begin{array}{l}\text { Desempeño } \\
\text { Docente }\end{array}$ & $\begin{array}{l}\text { Rendimiento } \\
\text { Académico }\end{array}$ \\
\hline \multirow{6}{*}{$\begin{array}{l}\text { Rho } \\
\text { de } \\
\text { Spearma } \\
\text { n }\end{array}$} & \multirow{3}{*}{$\begin{array}{l}\text { Desempeño } \\
\text { Docente }\end{array}$} & Coeficiente de correlación & 1,000 & $282^{* *}$ \\
\hline & & Sig. (unilateral) & . &, 003 \\
\hline & & $\mathrm{N}$ & 97 & 97 \\
\hline & \multirow{3}{*}{$\begin{array}{l}\text { Rendimiento } \\
\text { Académico }\end{array}$} & Coeficiente de correlación &, $282^{* *}$ & 1,000 \\
\hline & & Sig. (unilateral) &, 003 & . \\
\hline & & & & \\
\hline
\end{tabular}

**. La correlación es significativa en el nivel 0,01 (unilateral).

Fuente: elaboración propia.

En la tabla 7 se observan los estadísticos descriptivos de frecuencias de los Ítems 4, 5, 6, 7, 9 y 10, que participaron en la determinación de la correlación entre el Desempeño Docente y el Rendimiento Académico de los estudiantes. 
Tabla 7

Estadísticos descriptivos del Desempeño Docente

\begin{tabular}{|l|l|l|l|l|l|}
\hline Ítem & Nunca & Muy Poco & A veces & Siempre & \\
\hline 4. & 5 & $(\mathrm{f})$ & $(\mathrm{f})$ & $\mathrm{f})$ & \\
\hline 5. & 7 & 3 & 21 & 59 & 97 \\
\hline 6. & 2 & 6 & 24 & 63 & 97 \\
\hline 7. & 2 & 2 & 17 & 72 & 97 \\
\hline 9 & 4 & 5 & 14 & 79 & 97 \\
\hline 10. & 6 & 7 & 11 & 77 & 97 \\
\hline Total & 26 & 35 & 23 & 61 & 97 \\
\hline
\end{tabular}

Fuente: elaboración propia.

Con respecto a la Tabla 7 podemos deducir lo siguiente:

1. El $60,8 \%$ de los estudiantes afirma que el docente siempre genera confianza y absuelve las preguntas académicas de los estudiantes (ítem 4).

2. El $64,9 \%$ de los estudiantes afirma que el docente siempre propicia el aprendizaje significativo en las sesiones teóricas y prácticas (ítem 5).

3. El $74,2 \%$ de los estudiantes afirma que el docente siempre utiliza herramientas tecnológicas informáticas para la enseñanza de las asignaturas (ítem 6).

4. El $81,4 \%$ de los estudiantes afirma que el docente siempre utiliza estrategias metodológicas (videos, otras) para el proceso de enseñanza (ítem 7).

5. El 79,4\% de los estudiantes afirma que el docente siempre devuelve el instrumento de evaluación calificado (exámenes, otros) para su conocimiento (ítem 9).

6. El $62,9 \%$ de los estudiantes afirma que el docente siempre respeta las opiniones de los estudiantes (ítem 10). 
Con respecto a la Tabla 8 se observa los estadísticos descriptivos de frecuencias de las Notas o Calificaciones del Rendimiento Académico de los estudiantes.

\section{Tabla 8}

\section{Estadísticos descriptivos del Rendimiento Académico}

\begin{tabular}{|c|c|c|}
\hline Nota & (f) & $\%$ \\
\hline $0 \_10$ & 1 & 1,0 \\
\hline $11 \_13$ & 6 & 6,2 \\
\hline $14 \_16$ & 51 & 52,6 \\
\hline $17 \_19$ & 39 & 40,2 \\
\hline Total & 97 & 100,0 \\
\hline
\end{tabular}

Fuente: elaboración propia.

En la tabla 8 podemos observar lo siguiente:

1. El $1 \%$ de los estudiantes tiene un Rendimiento académico Bajo con una calificación vigesimal de 0 a 10 puntos de nota.

2. El 6,2\% de los estudiantes tiene un Rendimiento académico Regular con una calificación vigesimal de 11 a 13 puntos de nota.

3. El 52,6\% de los estudiantes tiene un Rendimiento académico Alto con una calificación vigesimal de 14 a 16 puntos de nota.

4. El 40,2\% de los estudiantes tiene un Rendimiento académico Muy Alto con una calificación vigesimal de 17 a 19 puntos de nota.

\section{Conclusión}

- Al analizar la correlación entre las variables Desempeño Docente y Rendimiento Académico (véase tabla 6), observamos que existe una correlación Positiva Baja de $\boldsymbol{R h o}=\mathbf{0 . 2 8 2}$, es decir, existe una asociación Positiva Baja de 28\% entre el Desempeño Docente y el Rendimiento Académico de los estudiantes de la Facultad de Ciencias de la Empresa de la UNAJMA.

- También podemos observar (véase tabla 6) que el p-valor =0,003 $<0,05$ y es significativa en el nivel 0,01(unilateral), esto indica que en la educación virtual sí hay una incidencia positiva del Desempeño Docente sobre el Rendimiento Académico de los estudiantes de la Facultad de Ciencias de la Empresa de la UNAJMA. 


\section{Referencias Bibliográficas}

Arias, F. G. (2012). El Proyecto de Investigación: Introducción a la Metodología de la Investigación. Caracas, Venezuela: Episteme.

Carrasco, S. (2005). Metodología de la Investigación Científica: Pautas Metodológicas para Diseñar y Elaborar el Proyecto de Investigación. Lima, Perú: San Marcos.

Cabañas, J. E. (2003). Aulas virtuales como herramienta de apoyo en la educación de la Universidad Nacional Mayor de San Marcos (tesis de pregrado). Universidad Nacional Mayor de San Marcos, Lima, Perú.

Camarena C. C. (2017). Estrategias de enseñanza virtual docente y su influencia en el rendimiento académico de los estudiantes del curso Desempeño Universitario en la Universidad Científica del sur, 2015. (tesis de maestría). Universidad Nacional Mayor de San Marcos, Lima, Perú.

Cepeda, L. G. (2017). Estrategia metodológica del uso de aulas virtuales en el proceso de enseñanza aprendizaje universitario. (tesis doctoral). Universidad Nacional Mayor de San Marcos, Lima, Perú.

Estrada, L. (2013). Importancia del desempeño docente. Universidad de Carabobo, Filosofía. Venezuela. Facultad de Ciencias de la Educación, 1-38. Recuperado de https://upload.wikimedia.org/wikipedia/commons/c/c5/EL_DESEMPE\%C3\%910_DO CENTE.pdf

Espinoza, E. (2006) Impacto del maltrato en el rendimiento académico. Revista electrónica de Investigación Psicoeducativa. N 9 Vol. 4 (2), 221-238. Recuperado de http://repositorio.ual.es/bitstream/handle/10835/638/Art_9_64.pdf?sequence=1

Hernández, R.; Fernández, C. \& Baptista, P. (2014). Metodología de la investigación. C. D. México, México: Mc Graw Hill.

Montenegro, I. A. (2007). Evaluación del desempeño docente: fundamentos, modelos e instrumentos. Bogotá, Colombia: Cooperativa Editorial Magisterio.

Ministerio de Educación de Colombia (2020). Educación virtual o educación en línea. Colombia. Recuperado de

https://www.mineducacion.gov.co/1759/w3-article-196492.html?_noredirect=1 
Reyes, Y. (2003). Relación entre el rendimiento académico, la ansiedad ante los exámenes, los rasgos de personalidad, el autoconcepto y el asertividad en estudiantes del primer año de psicología de la UNMSM. (tesis doctoral). Universidad Nacional Mayor de San Marcos, Lima, Perú.

UNESCO/IESALC (2003) La Educación Superior Virtual en América Latina y el Caribe. Instituto Internacional para la Educación Superior en América Latina y el Caribe. Recuperado de http://hdl.handle.net/123456789/527 